The development of the social aspect of the world community is closely related to the expansion of the range of digital services in cyberspace. A special place in which social networks occupy. The world's leading states are conducting information operations in this environment to achieve geopolitical goals. Such processes are reflected in real social and political life. This makes it possible to influence not only the social groups of society, but also to ensure manipulation in political "games" in the conduct of hybrid wars.

The simultaneous interaction of social factors, influencing factors, the presence of communities in social networks forms a full-fledged sociocyber-physical system capable of integrating real and virtual interactions to manage regional communities.

The article proposes a method for predicting the assessment of social mutual influence between "formal" and "informal" leaders and regional societies. The proposed models make it possible to form not only a forecast of the influence of agents, but also the interaction of various agents, taking into account their formal and informal influences, the use of administrative resources, political moods of the regional society. This approach allows dynamic modeling based on impact and relationship analysis.

The presented results of simulation modeling do not contradict the results of opinion polls and make it possible to form a set of measures that can be aimed at overcoming the negative impact on the regional society of both individual "leaders" and political parties. Analysis of the simulation results allows to increase both the political and social stability of the regional society, helps to prevent conflict moods and contradictions

Keywords: socio-cyber-physical system, social networks, models of influence, rating of political parties, regional society
UDC 004.056:004.738.5(045)

DOI: $10.15587 / 1729-4061.2021 .249313$

\section{DEVELOPMENT OF A METHOD FOR ASSESSING FORECAST OF SOCIAL IMPACT IN REGIONAL COMMUNITIES}

\author{
Serhii Yevseiev \\ Corresponding author \\ Doctor of Technical Sciences, Professor* \\ E-mail: serhii.yevseiev@hneu.net \\ Yurii Ryabukha \\ Doctor of Technical Sciences, Senior Researcher \\ Department of Information Systems and Technologies Security \\ V. N. Karazin Kharkiv National University \\ Svobody sq., 4, Kharkiv, Ukraine, 61022 \\ O leksandr Milov \\ Doctor of Technical Sciences, Professor* \\ Stanislav Milevskyi \\ $\mathrm{PhD}$, Associate Professor* \\ Serhii Pohasii \\ $\mathrm{PhD}$, Associate Professor* \\ Yevheniia Ivanchenko \\ $\mathrm{PhD}$, Professor** \\ I hor Ivanchenko \\ $\mathrm{PhD}$, Associate Professor** \\ Yevgen Melenti \\ $\mathrm{PhD}$
}

Special Department No. 2 «Tactical-Special Training, Marksmanship Training and Special Physical Training» Juridical Personnel Training Institute for the Security Service of Ukraine

Yaroslav Mudryi National Law University Myronosytska str., 71, Kharkiv, Ukraine, 61002

Ivan Opirsky y

Doctor of Technical Sciences Department of Information Security Lviv Polytechnic National University S. Bandery str., 12, Lviv, Ukraine, 79013

I gor Pasko

$\mathrm{PhD}$, Senior Researcher Scientific-Research Center of Missile Troops and Artillery Herasima Kondratieva str. 165, Sumy, Ukraine, 40021 *Department of Cyber Security and Information Technology Simon Kuznets Kharkiv National University of Economics Nauky ave., 9-A, Kharkiv, Ukraine, 61166 **Department of Information Technology Security National Aviation University Liubomyra Huzara ave., 1, Kyiv, Ukraine, 03058
How to Cite: Yevseiev, S., Ryabukha, Y., Milov, O., Milevskyi, S., Pohasii, S., Ivanchenko, Y., Ivanchenko, I., Melenti, Y., Opirskyy, I., Pasko, I. (2021). Development of a method for assessing forecast of social impact in regional communities. Eastern-European Journal of Enterprise Technologies, 6 (2 (114)), 30-43. doi: https://doi.org/10.15587/1729-4061.2021.249313

\section{Introduction}

In the modern world in the context of globalization, the Internet is becoming the main information technology and social form, a universal mean of free communication and information retrieval. The transition of social communication to the virtual sphere has intensified due to the global situation related to the COVID-19 pandemic. It is in the virtual environment that the maximum of social interaction takes place, the construction of interpersonal connections and networks, the implementation of which became possible thanks to self-governing network communication based on personal choice. 
In the context of the formation of a high-tech society, social networks based on Internet services have become one of the most effective and popular means of mass communication. Influence on such communities is an effective mechanism of impact in the context of hybrid wars and color revolutions [1]. Such a synthesis of social Internet services (SIS) with cyber-physical systems makes it possible to form a cyber-physical social system (CPSS) [2-6]. CPSS allow to form social, political, economic "opinion" of the intellectual community (integration of the cybernetic, physical and social worlds), to regulate and manage on the basis of the SIS, to provide users with proactive services. The nature of CPSS data brings new requirements and challenges to the various stages of data processing, including identifying data sources, processing and combining data of various types and scales.

Achievements in the field of information technology were a prerequisite for the creation of a new form of social groups, called "virtual communities", the influence on which can allow achieving the necessary target states or the reactions of such communities. The virtual community is a reflection of the connections, relationships and interactions of people taking place in social life, but every day they are more and more regularly transferred to free and boundless cyberspace.

The identification of such communities and groups in the network and the identification of the most influential agents will determine the degree and direction of the necessary social influences to achieve the goals set.

The integration of CPSS with "virtual communities" greatly influences the tasks of forming both political, social and economic worldviews. The latter can be formed on the basis of the influence of both state subjects of government, political parties, and informal leaders of the regional community. The block diagram of the CPSS is shown in Fig. 1.
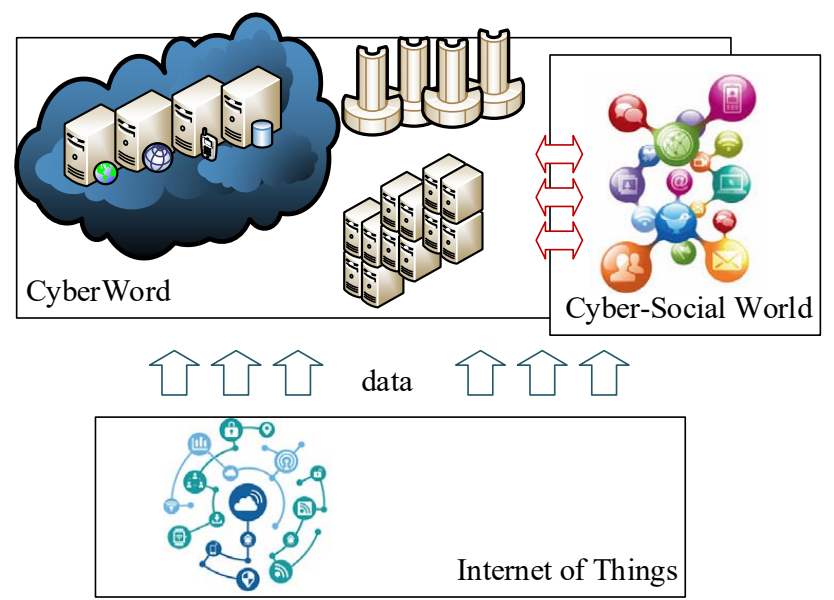

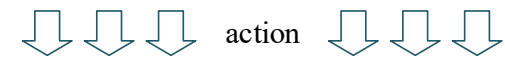

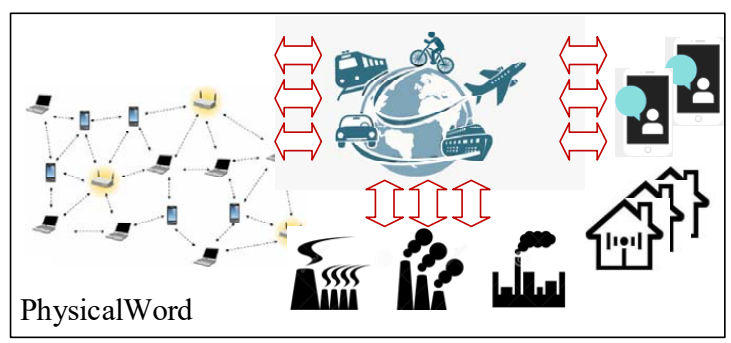

Fig. 1. Block diagram of the interaction of CPSS elements
A variety of devices that can be used in integrated information and communication systems (ICS) and cyber-physical systems (CPS) make it possible to form the concept of a socio-cyber-physical system (CPSS) with the SIS. CPSS is a set of subjects and objects of the cybernetic, physical and social worlds, which make it possible to form "smart" communities, on the one hand, and intellectual space, on the other. In CPSS, users are service consumers, and physical entities in the form of various devices are service providers [2].

Thus, the integration of the cybernetic, physical and social worlds allows the creation of smart communities, that is, those capable of behaving rationally. From a social point of view, smart communities can promote social awareness among members using certain social sensors [2].

To study the features of the creation and development of virtual (smart) communities, specialized research organizations have been created. Among them are such as "Communication Institute for Online Scholarship", "The UCLA Center for the Study of Online Community", "Association of Internet Researchers", "International Society for Mental Health Online", "The Society for Computers in Psychology" [7]. The results of the work of such organizations are of particular importance for structures seeking to establish their presence in the electronic environment, as well as for scientists trying to understand the behavior of Internet users. However, despite the fact that such studies have been carried out for quite a long time, at the moment the mechanisms and methods of purposeful influence on social communities have not been sufficiently developed, which makes it possible to judge the prospects of the stated research topic.

Thus, CPSS allow not only to form and develop the functions of a smart community and intellectual space, but also to influence the behavior of communities through the SIS, ensuring the formation of a predictable worldview. Assessment of the impact on social groups in the regional community in this aspect affects the national security of the state as a whole, which confirms the relevance of this area of research.

\section{Literature review and problem statement}

In a globalized, interconnected and data-driven world, people increasingly rely on online services to meet their needs. Such services typically use feedback and reputation mechanisms to evaluate their products, services, vendors, and customers. Ideas and opinions are increasingly disseminated through social media, to the point that they can trigger political shifts [7]. In this context, it is very important to understand how social influence influences individual decision-making and how it affects the group level.

In [8], a model is presented that allows to quantify how social information affects individual assessments and opinions. The impact of social information at the individual and collective levels was measured and modeled on assessment tasks with low evidence. The influence of virtual experts on the work of the group was also studied. As a result, the conclusion was formed: when experts provide the group with reliable information, a systematic improvement in collective accuracy is achieved experimentally and quantitatively reproduced by the model.

However, work [8] does not consider aspects related to identifying such "experts" and assessing the strength and reliability of their influence on social groups. 
The category "Wisdom of the crowd" is considered as an example and variant of social influence. In [9], it was proved that networks of dynamic influence can adapt to biased and non-stationary conditions, prompting individual and collective beliefs to become more accurate than the independent beliefs of the most successful people.

But at the same time, the mechanisms of the influence of individuals on common collective beliefs have not been sufficiently disclosed.

In work [10], the distribution of physical quantities through social networks is considered. The spreading phenomenon has been studied by constructing a mathematical model. The model can track changes in attribute values in the propagation engine. Given the certain network relationships between players, the token donation game is conducted as a physics experiment to collect data that are analyzed to build a mathematical model. Model-based computer experiments are carried out to study the rates of diffusion and decay, which determine, respectively, the direction of flow and the decrease in magnitude towards steady state.

At the same time, the application of such a model to study the information impact on social groups is difficult due to the difficulty of determining the physical equivalent of the information impact.

The article [11] examines the effect of error leading to a change in the "Wisdom of the crowd" under the influence of individual and social factors. Moreover, the individual impact is taken into account as a systematic error.

In addition, the rules of social influence and the effect of social bias have been empirically determined. This asymmetric effect suggests that the target person was more strongly influenced by social information that was more significant compared to the focus person's rating, compared to social information that was less than the individual's rating. An observed increase in the coefficient of variation with increasing numbers may indicate that self-confidence decreases as numbers increase, which could lead to an asymmetric effect of social bias.

However, the search strategy for "hidden experts" [11] in this study did not yield any results. Finding hidden experts requires additional information about people (for example, propensity to use social information, past success, or confidence level). The method developed by the authors [11] does not require any additional information about each person, only knowledge about the statistical trends of the population as a whole. And thus, the mechanism of targeted individual impact on the behavior of social groups is not disclosed.

The article [12] presents the results of a social experiment in which it is proved that, based on the effect of the wisdom of the crowd, groups can be extremely accurate in assessing little-known facts. From the point of view of decision-makers, it would be helpful to solicit several independent opinions and combine them as a basis for their judgments. It is hardly possible to get an independent opinion in society, because people are embedded in social networks and usually influence each other to a certain extent.

Experimental results [12] show that social influence causes convergence of individual assessments and significantly reduces the diversity of the group without increasing its accuracy. The remaining variety is often so small that the correct value shifts from the center to the outer regions of the rating range. Thus, by making committee decisions or following the advice of a socially influenced expert group, their opinions can lead to a set of predictions that no longer even contain the correct meaning. From the point of view of decision-makers, such advice can be completely misleading, since closely related, seemingly independent advice can claim certainty, despite significant deviations from the correct decision.

However, the study did not examine the influence of the group leader, belief, or any other socio-psychological influence.

In the article [13], an agent-based model was built using empirical distributions of personal assessments and distributions of sensitivity to social influence, as well as the effect of distance. The model was used to generate forecasts for large groups and a number of overall estimates. The model predicts that the improvement in individual accuracy prevails over collective accuracy and is higher with aggregate processing than with both other schemes. It was found that in small groups, the improvement in accuracy is the same when all estimates are used together or their geometric mean. However, people use different strategies to improve their grades. An empirical model was used to generate predictions for larger populations. Sharing averages is expected to be better than sharing complete information in improving the accuracy of estimates in larger groups.

It should be noted that in this work, as in many others, an approach to assessing the degree of individual target influence on social groups was not developed.

In [14], it was proposed to extract information from the collective using those people who resist social influence. Methods for extracting information that the team considers confidential are proposed. The idea that different people or subgroups of people have different biases is consistent with the existence of different procedures in a population for solving a problem, each with a different bias. A second and additional explanation for personality is that people have different levels of knowledge in a subject or even in general assessment exercises. This level of knowledge is probably not high enough for people to claim it, but it will be enough to act on it when faced with social influence. The methods proposed for improving the "wisdom of the crowd" do not correspond to the usual situation in which people interact naturally. On the contrary, it is a protocol that can be used to extract high quality information in human collectives, even if it is only present in a minority of the group.

The analysis of works devoted to the identification of multidirectional influence in social and virtual communities [8-14] showed that there are no methods for assessing the influence of both "formal" and "informal" leaders, political ties on a regional society, or an individual. Also in the cited works [8-14] more attention is paid to the influence of collective opinion on the individual, but not vice versa.

The need to develop a method for assessing social influence is due to the actual presence of such influences, especially in situations related to elections and assessing the influence of individual agents on the positions and ratings of political forces.

\section{The aim and objectives of research}

The aim of research is to develop a method for assessing social impact in regional communities. The method is based on matrix models of the interaction of network agents, taking into account the exposure to the influence of various government agencies and organizations, while taking into 
account the political activity of the participants in the process. This approach will make it possible to timely receive a dynamic change in the level of exposure to social influence, in offline mode to form targeted impacts based on the calculated characteristics.

To achieve the goal of the work, it is necessary to solve the following tasks:

- to develop models for assessing the social influence of state institutions, media and informal leaders on regional communities, their attitude to political parties, the influence of a regional society on the formation of a rating of political forces;

- to develop a method for assessing the total intensity of the influence of a particular institutional structure;

- to develop a method for assessing and predicting the rating of political forces based on the mechanism of social influence.

\section{Materials and methods of research}

One of the main points in determining the impact on a community and a social group is the very selection of this community within the entire social network or society as a whole.

A community is defined as a group of nodes with tighter internal connections than with the rest of the network [15]. This intuitive definition has been formalized in several competing ways, usually as a quality function that quantifies the quality of a given division of the network into communities. The main approaches to isolating a community from a network are shown in Fig. 2. graph, normalized to the total weight of the edges $S$ and the rest of the graph $\bar{S}$. It is intuitively clear that groups with a low normalized cut are good communities because they are well connected with each other, but weakly connected with the rest of the graph.

Kernighan-Lin algorithm (KL). A vertex is not considered re-moved if it has already been moved in the current iteration. After a vertex has been moved, the increment for its adjacent vertices will be updated to reflect the new assignment of vertices to partitions. Although each iteration in the original KL algorithm [15] had the complexity $O(|E| \log |E|)$, further improved to $O(|E|)$ per iteration using the appropriate data structures. This algorithm can be extended to multiple separate sections, enhancing each pair of sections in a multi-user section in the manner described above.

Agglomerative/dividing algorithms [15] start at each node of a social network in its own community, and at each stage combine communities that are considered to be reasonably similar. This continues until either the desired number of communities is obtained, or the remaining communities are not too dissimilar to further merge. Partitioning algorithms work in reverse; they start with the whole network as one community and at each stage they choose a specific community and split it into two parts. Both types of hierarchical clustering algorithms often output a dendrogram, which is a binary tree where the leaves are the nodes of the network and each inner node is a community. In the case of dividing algorithms, the parent-child relationship indicates that the community represented by the parent node has been split to get the communities represented by the child nodes. In the case of agglomerative algorithms, the parent-child relationship in the dendrogram indicates that the communities represented by the child nodes have been agglomerated (or merged) to get the community represented by the parent node.

Spectral algorithms belong to the classical methods of clustering and community detection. Spectral techniques generally refer to algorithms that assign nodes to communities based on eigenvectors of matrices, such as the adjacency matrix of the network itself or other related matrices. The up-
The features of each of the methods are discussed below. Quality functions.

In the literature [14, 15], many functions or quality indicators have been proposed to reflect the quality of dividing a graph into clusters. Hereinafter, A denotes the adjacency matrix of a network or graph, where $A(i, j)$ represents the edge weight or affinity between nodes $i$ and $j$, and $V$ denotes a vertex or a set of nodes of the graph or network.

Normalized section of a vertex group $S \subset V$ defined as:

$$
N_{\text {cut }}(S)=\frac{\sum_{i \in S, j \in \bar{S}} A(i, j)}{\sum_{i \in S} \operatorname{degree}(i)}+\frac{\sum_{i \in S, j \in \bar{S}} A(i, j)}{\sum_{i \in S} \operatorname{degree}(j)},
$$

where the normalized cut of a group of nodes $S$ - the sum of the weights of the edges that connect $S$ to the rest of the per $k$ eigenvectors define the nesting of the hosts as points in k-dimensional space, and then classical data clustering techniques such as K-means clustering can be used to get the final assignment of the nodes to the clusters [15]. The main idea behind spectral clustering is that the low-dimensional representation induced by the upper eigenvectors reveals the structure of the cluster in the original graph with greater clarity.

Multilevel methods provide a powerful framework for fast and high-quality graph partitioning, and in fact they have also been used to solve many other problems [15]. The main idea is to sequentially shrink or enlarge the input graph to get a small graph. Then split this small graph and sequentially project this split back onto the original graph, refining the split at each step.

Steen van Dongen's Markov Clustering Algorithm (MCL) clusters graphs by manipulating the stochastic matrix or transition probability matrix corresponding to the graph [15]. 
In what follows, the probability of a transition between two nodes is also called stochastic flow. The MCL process consists of two operations on stochastic matrices: Expand и Inflate. Expand $(M)$ - it's just $M M$, and Inflate $(M, r)$ increases each entry in the matrix $M$ to the inflation parameter $r$ ( $>1$ and is usually installed as 2$)$ and then re-normalizing the columns to sum to 1 . These two operators are applied alternately iteratively until convergence, starting with the original transition probability matrix.

The considered methods of identifying communities in a social network are quite effective. However, they can only be used with a relatively small number of members and networks and communities. Scaling these methods to a real social network extremely complicates the processes of calculations and identification of communities.

In addition to defining the boundaries and participants of social communities, it is also necessary to determine the type and nature of social influence on the behavior of such communities.

The central issue of social influence is understanding the relationship between similarities and social connections [16]. Many studies have attempted to measure social media influence and correlation from a wide variety of perspectives. Such aspects are social similarity and influence; social impact marketing, impact maximization; the model and practice of social influence through conformity, compliance and obedience, as well as social influence in virtual worlds.

The presence of social influence can be determined using traditional methods.

Homophilia [16] is one of the most fundamental characteristics of social networks. This suggests that the actor on the social network tends to resemble their connected neighbors or "friends". This is a natural result, because a given actor's friends or neighbors on a social network are not a random sample from the general population.

Existential Social Impact Test.

In [17], the authors try to separate social influence from internal or interfering variables by proposing a shuffle test and a back edge test. The idea behind the random test is that if social influence is not important, the timing of such activation should not depend on the duration of the action of other agents. Even though the likelihood of an agent activating may depend on his/her friends. Therefore, the data distribution and characteristics will not change even if the exact time of occurrence is changed. The idea behind the edge-shifting test is that other forms of social correlation (besides social influence) are based only on the following. Two friends often share common characteristics or are influenced by the same external variables. Thus, changing the margins will not significantly change the score for social correlation. On the other hand, social influence extends in the direction indicated by the edges of the graph, and therefore changing the direction of the edges should intuitively change the correlation score.

[18] tests models using tag data from Flickr and confirms social influence as a source of correlation between the actions of socially connected people.

Influence and action. Influence is usually reflected in changing patterns of social action (user behavior) on a social network. In the works $[18,19]$, the problem of studying the degree of influence on the basis of the user's historical actions was studied. Other works [20,21] explore how social actions develop in the context of a network and how they are influenced by social influence.
Influence and interaction. In addition to the attribute and user actions, influence can also be reflected in the interactions between users [19]. Usually, online communities contain additional information about interaction with users. For example, a Facebook user has a wall page where his/her friends can post. According to the messages posted on the Wall, it can be concluded which friends are close, and which are only acquaintances. Likewise, it is possible to use Twitter followers and followers to infer the strength of a relationship.

Maximum impact in viral marketing. Social impact analysis has many practical applications. Impact maximization in viral marketing is an example of such an important application [19]. The problem is often motivated by identifying leads for marketing purposes. The goal is to minimize marketing costs and more generally to maximize profits. For example, a company may want to sell a new product through the natural word-of-mouth effect that results from interactions on a social network. The goal is to attract a small number of influential users to product adoption and subsequently trigger a large cascade of further adoption. To achieve this goal, a measure is needed to quantify the intrinsic characteristics of the user (for example, the expected profit from the user) and the network value of the user (for example, the expected profit from the users).

Thus, social influence analysis aims to qualitatively and quantitatively measure the influence of one person on others. As social media becomes more prevalent in the daily activities of millions of people, both research and practical applications on social impact will continue to grow. In addition, the size of the networks in which the underlying applications are to be used also continues to grow over time. Therefore, effective methods of social impact are in demand.

The proposed method for assessing social influence in regional communities is based on matrix models of interaction between network agents, taking into account the exposure to the influence of various government institutions and organizations, while taking into account the political activity of the participants in the process. This approach allows to get a dynamic change in the level of exposure to social influence in a timely manner. And also, to form not only a forecast of the influence of agents, but also the interaction of various agents, taking into account their formal and informal influences, the use of administrative resources, political moods of the regional society. Thus, the final sequence of steps makes it possible to significantly simplify the obtaining of integrated results of the political and social situation at the regional level.

\section{Results of the development of a method for assessing social impact in regional communities}

\section{1. Development of models for assessing the impact of formal and informal leaders on regional communities}

Mathematical models for assessing the susceptibility to social influence of regional communities from the point of view of attitudes towards political parties can be formally set in matrix form. The influence of elements of state institutions, media and informal leaders, and the regional society on the formation of the rating of political forces is set in a similar way.

Let's introduce into consideration the following sets of elements and their characteristics:

- $A A=\left\{A A_{1}, A A_{2}, \ldots, A A_{k}\right\}$ - set of state institutions of power (formal leaders). For the convenience of subsequent 
calculations, let's represent the set in the form of a one-dimensional vector $\mathbf{A}=\left(A A_{1}, A A_{2}, \ldots, A A_{k}\right)$. For each of the elements, the level of the organizational and state hierarchy is determined, which this element occupies. For each of the levels, let's define the weight coefficient $\lambda$, which takes into account the "power weight" (political weight) of the hierarchical level of the elements of state institutions. So, with a four-level model of state structure, the values of the weighting coefficient of the level are defined as $\lambda_{i}\{1,0.75$, $0.5,0.25\}$. Thus, the higher the level of the state hierarchy occupied by this or that element, the more significant its political influence on the regional society is supposed to be (begin the numbering of levels from the highest);

- $P P=\left\{P P_{1}, P P_{2}, \ldots, P P_{n}\right\}$ - set of political forces (parties, blocs, movements, political parties), presented as a one-dimensional vector $\mathbf{P}=\left(P P_{1}, P P_{2}, \ldots, P P_{n}\right)$; each political force can be assigned a weight coefficient reflecting its rating $-\theta_{i} \in[0,1]$;

- IL $=\left\{I L_{1}, I L_{2}, \ldots, I L_{l}\right\}$ - set of informal leaders of the regional community, which include: heads of enterprises, organizations, companies, criminals, cyber intruders, etc., presented as a one-dimensional vector;

$-M M=\left\{M_{1}, M_{2}, \ldots, M_{m}\right\}$ - set of elements of the media (media), which include: mass media (media, newspapers and magazines of the central and local level). Internet (social networks, media resources), television, radio, presented as a one-dimensional vector $\mathbf{M}=\left(M_{1}, M_{2}, \ldots, M_{m}\right)$;

- $S S=\left\{S S_{1}, S S_{2}, S S_{3}, S S_{4}\right\}$ - regional community (society), represented by set of its age groups (segments of society). The division into age groups is standard for sociology and is determined by the following age ranges (in years) (17-30), (31-60), (61-75), (76-90). Each age category of a regional society must be matched with two coefficients. The coefficient of political activity, which can be considered as involvement in social processes and, therefore, susceptibility to social manipulation $-\Psi_{i} \in\{0.75,1,0.5,0.25\}$. And the share of the corresponding age category in the total number of persons making up the regional society $\mathbf{W}=\left(w_{1}, w_{2}, w_{3}, w_{4}\right)$.

The influence of relevant individuals and organizations (sets $A A, P P, I L, M M$ ) on various categories of regional society (set $\mathrm{SS}$ ) can be formally represented by the matrix IMP. Matrix size $(k+l+m+n) \times 4$, where $m$ - the cardinality of the set $M M, l-$ is the cardinality of the set $I L$, and $k-$ is the cardinality of the set $A A, n$ - is the cardinality of the set $P P$.

As matrix elements are used values $\mu_{i j} \in\{1,0.75,0.5$, $0.25\}$, which are considered as weights reflecting the strength of the social influence of elements of state institutions, media and informal leaders on the attitude of regional communities towards political parties. Wherein $\mu_{i j}$ can be both positive and negative (negative value denotes the negative influence of elements of state institutions, media and informal leaders on the attitude of regional communities towards political parties).

The structural diagram of the interaction between the subjects of the regional society and formal and informal leaders is shown in Fig. 3.

Let's form influence matrices for various sets that form the basis of the developed models.

Step 1. Let's form a matrix of distribution of state institutions (formal leaders) by levels of the organizational-state hierarchy:

$$
H=\left(\begin{array}{ccc}
h_{11} & \ldots & h_{14} \\
\ldots & \ldots & \ldots \\
h_{k 1} & \ldots & h_{k 4}
\end{array}\right) .
$$

The number of matrix rows corresponds to the number of state institutions (formal leaders), and the number of columns corresponds to the number of levels of the public administration system (in this case, it is assumed to be 4). Element $h_{i j}$ is equal to 1 if the state institution $i$ is at the level $j$, and $0-$ otherwise. Since it is possible for each institution to be at only one level, then for the formed matrix, a system of restrictions can be written:

$$
\left\{\begin{array}{l}
h_{i j} \in\{0,1\} i=\overline{1, k}, j=\overline{1,4}, \\
\sum_{j=1}^{4} h_{i j}=1 .
\end{array}\right.
$$

It is assumed that for state institutions their political weight (that is, the importance of the expressed opinion, point of view) is the greater, the higher the level of the organizational-state hierarchy (as a reflection of political weight). Consequently, the previously obtained values of the coefficients $a_{i j}$, based on the distribution over the levels of the hierarchy, should be adjusted, and will be calculated as follows:

$$
h_{i j}^{\prime}=h_{i j} \times \lambda_{j}, \quad i=\overline{1, k}, \quad j=\overline{1,4}, \quad \lambda_{j} \in\{1,0.75,0.5,0.25\} .
$$

The political weight of the respective institution is determined as follows:

$$
v_{i}=\sum_{j=1}^{4} h_{i j}^{\prime}
$$

Formation of the vector of weight coefficients of influence on the regional society of state institutions, taking into account their hierarchical level:

$$
\eta_{i j}=v_{i} \times \mu_{i j}
$$

Step 2. After the formation of the value of the political weight of formal leaders, it is possible to form a matrix of the influence of the elements of state institutions of power, depending on the levels of the hierarchical model of the state:

$$
h_{i j}^{\prime \prime}=h_{i j}^{\prime} \times \eta_{i j} .
$$

Similar adjustments to the coefficients of influence of informal leaders and mass media can be made for the corresponding sets. However, at this stage of building the model of influence, these adjustments will not be performed and can be postponed to later stages of adjusting the model.

Step 3. Formation of the vector of weight coefficients of political activity of age groups of the regional society, taking into account the share of the corresponding age category in the total size of the regional society:

$$
\sigma_{i}=w_{i} \times \psi_{i}, \quad i=\overline{1,4}
$$

where $w_{i}=\frac{N_{i}}{N_{0}}, N_{0}-$ total size of the territorial community; $N_{i}$ - the number of the corresponding age category of the territorial community. 


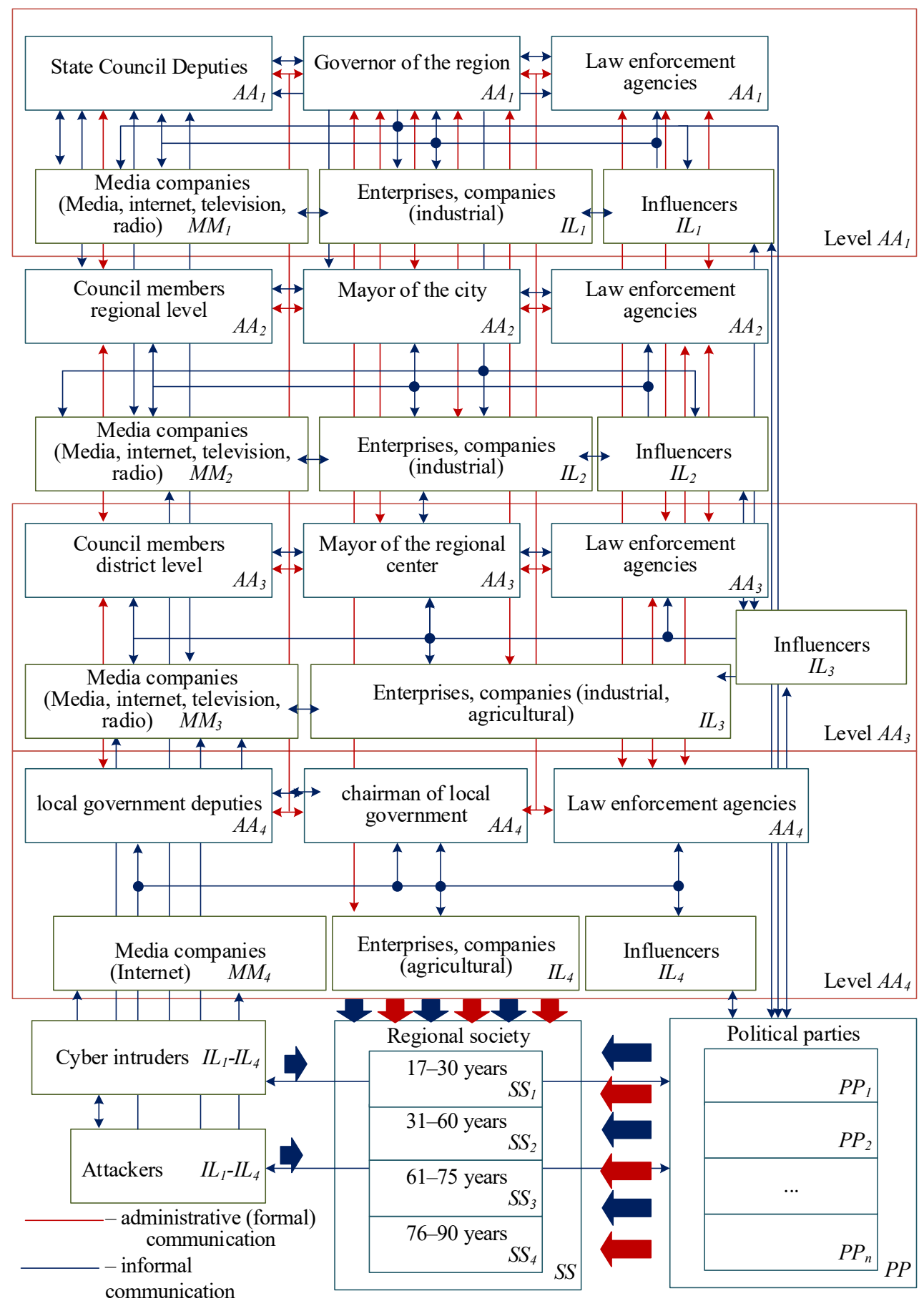

Fig. 3. Structural diagram of interaction between the subjects of regional society and formal and informal leaders

Step 4. Formation of matrices of influence of formal and informal leaders, mass media, media, political parties on the regional society, taking into account its age structure, will be represented by the matrix IMP. Matrix dimension $(k+l+m+n) \times 4$, where $k, l, m, n$ were previously defined as the cardinalities of sets AA, IL, MM, PP. As elements of matrix are used sets $\mu_{i j} \in\{1,0.75,0.5,0.25\}$. They are considered as the weighting coefficients of the social influence of the $i$-th element of the set of state institutions, media and informal leaders on the attitude of the $j$-th age group of regional communities to political parties. Wherein $\mu_{i j}$ can be both positive and negative (negative value denotes the negative influence of elements of state institutions, media and informal leaders on the attitude of regional communities towards political parties).

Influence of elements of the corresponding sets AA, MM, IL and $\mathrm{PP}$ on different age groups of the regional community are reflected in the corresponding matrices that form a generalized matrix of influence:

- for formal leaders:

$\mathbf{A A}=\left(\begin{array}{ccc}\eta_{1,1} & \ldots & \eta_{1,4} \\ \ldots & \ldots & \ldots \\ \eta_{k, 1} & \ldots & \eta_{k, 4}\end{array}\right)$

- for informal leaders: 
$\mathbf{I L}=\left(\begin{array}{ccc}\mu_{1,1} & \ldots & \mu_{1,4} \\ \ldots & \ldots & \ldots \\ \mu_{l, 1} & \ldots & \mu_{l, 4}\end{array}\right)$

- for the media:

$\mathbf{M M}=\left(\begin{array}{ccc}\rho_{1,1} & \ldots & \rho_{1,4} \\ \ldots & \ldots & \ldots \\ \rho_{m, 1} & \ldots & \rho_{m, 4}\end{array}\right) ;$

- for political parties:

$\mathbf{P P}=\left(\begin{array}{ccc}\theta_{1,1} & \ldots & \theta_{1,4} \\ \ldots & \ldots & \ldots \\ \theta_{k, 1} & \ldots & \theta_{k, 4}\end{array}\right)$.
A mathematical model for assessing the susceptibility to social influence of elements of state institutions, media and informal leaders on regional communities from the point of view of attitudes towards political parties is formally set and presented in Fig. 4:

$$
\mathbf{I M P}=\mathbf{A A} \cup \mathbf{I L} \cup \mathbf{M M} \cup \mathbf{P P},
$$

where the matrix IMP - generalized matrix of the influence of various institutions on the corresponding age groups of the regional community.

Matrix IMP is formed by appending the rows of the next matrix to the existing one. As a result, will be formed a matrix with the dimension $(k+l+m+n) \times 4$. In it, lines in the range from 1 to $k$ correspond to formal leaders.

Initial data

$A A=\left\{A A_{1}, A A_{2}, \ldots, A A_{k}\right\}$ - set of government institutions;

$\lambda_{i} \in\{1,0.75,0.5,0.25\}$ - government level weighting factor;

$P P=\left\{P P_{1}, P P_{2}, \ldots, P P_{n}\right\}-$ set of political forces;

a weighting factor reflecting its rating $-\theta i \in[0,1]$;

$I L=\left\{I L_{1}, I L_{2}, \ldots, I L_{l}\right\}-$ set of informal leaders of the regional society;

$M M=\left\{M_{1}, M_{2}, \ldots, M_{m}\right\}-$ set of media elements;

$S S=\left\{S S_{1}, S S_{2}, S S_{3}, S S_{4}\right\}$ - regional community (society), with age ranges (in years) (17-30), (3160), (61-75), (76-90);

$\psi_{i} \in\{0.75,1,0.5,0.25\}$ - coefficient of political activity;

$\mu_{i j} \in\{1,0.75,0.5,0.25\}$ - weight coefficients reflecting the strength of the social influence of elements of state institutions, media and informal leaders on the attitude of regional communities towards political parties
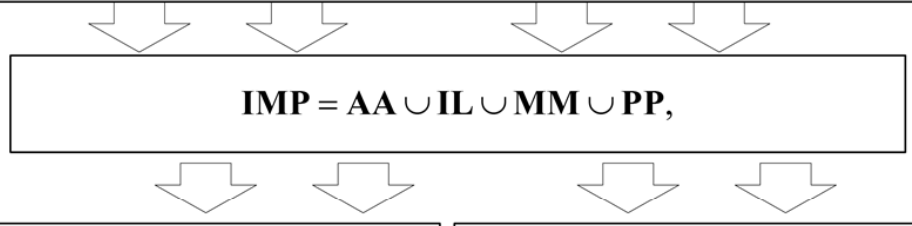

Determination of the total intensity of the influence of the institutional structure:

additive convolution:

$$
\omega_{i}=\sum_{j=1}^{4} \rho_{i j},
$$

multiplicative convolution:

$$
\omega_{i}=1-\sum_{j=1}^{4}\left(1-\rho_{i j}\right),
$$

where

$\rho_{i j}=\mu_{i j} \times \sigma_{j}, \sigma_{i}=w_{i} \times \psi_{i}, \quad i=\overline{1,4}, w_{i}=\frac{N_{i}}{N_{0}}$, $N_{0}$ - total size of the territorial community;

$N_{i}$ - the number of the corresponding age category of the territorial community

Determination of the total intensity of influence on age groups:

additive convolution:

$$
\tau_{j}=\sum_{i=1}^{k+l+m+n} \rho_{i j}
$$

multiplicative convolution:

$$
\tau_{j}=1-\sum_{i=1}^{k+l+m+n}\left(1-\rho_{i j}\right)
$$

In the range from $k+1$ to $k+l$ - informal, ranging from $k+l+1$ to $k+l+m$ - media, and ranging from $k+l+m$ to $k+l+m+n$ - political forces.

Fig. 4. Mathematical model for assessing exposure to social influence 
In the range from $k+1$ to $k+l$ - informal, ranging from $k+l+1$ to $k+l+m$ - media, and ranging from $k+l+m$ to $k+l+m+n-$ political forces:

$$
\begin{aligned}
& \mathbf{I M P}=\left(\begin{array}{c}
\mathbf{A A} \\
\mathbf{I L} \\
\mathbf{M M} \\
\mathbf{P P}
\end{array}\right)= \\
& =\left(\begin{array}{lll}
\eta_{11} \cdot \sigma_{1} & \ldots & \eta_{14} \cdot \sigma_{4} \\
\ldots & \ldots & \ldots \\
\eta_{k 1} \cdot \sigma_{1} & \ldots & \eta_{k 4} \cdot \sigma_{4} \\
\mu_{k+1,1} \cdot \sigma_{1} & \ldots & \mu_{k+1,1} \cdot \sigma_{4} \\
\ldots & \ldots & \ldots \\
\mu_{k+l, 1} \cdot \sigma_{1} & \ldots & \mu_{k+l, 1} \cdot \sigma_{4} \\
\rho_{k+l+1,4} & \ldots & \rho_{k+l+1,4} \\
\ldots & \ldots & \ldots \\
\rho_{k+l+m, 4} & \ldots & \rho_{k+l+m, 4} \\
\theta_{k+l+m+1,1} \cdot \sigma_{1} & \ldots & \theta_{k+l+m+1,4} \cdot \sigma_{4} \\
. . & \ldots & \ldots \\
\theta_{k+l+m+n, 1} \cdot \sigma_{1} & \ldots & \theta_{k+l+m+n, 4} \cdot \sigma_{4}
\end{array}\right) .
\end{aligned}
$$

Thus, the developed mathematical models make it possible, on the basis of an expert assessment, to obtain an objective reflection of the influence of individual CPSS groups, their relationship and influence on the regional society. For the correct assessment of experts, let's use the mathematical apparatus proposed in [22].

\section{2. Development of a method for assessing the total in-} tensity of the influence of a particular institutional structure

The method for assessing the total intensity of influence is formed on the basis of a mathematical model for as- sessing the susceptibility to social influence of elements of state institutions, media and informal leaders on regional communities and the corresponding convolution.

The elements of the resulting matrix are calculated taking into account the political activity of different age groups:

$$
\rho_{i j}=\mu_{i j} \times \sigma_{j},
$$

Step 5. The calculation of the total intensity of the influence of a particular institutional structure (formal or informal leader, political party, mass media) can be presented as a convolution by row (for all age categories):

$$
\begin{aligned}
& \text { - for additive convolution }-\omega_{i}=\sum_{j=1}^{4} \rho_{i j} ; \\
& \text { - for multiplicative convolution }-\omega_{i}=1-\sum_{j=1}^{4}\left(1-\rho_{i j}\right) .
\end{aligned}
$$

Step 6. Similarly to the 5th step, the calculation of the intensity of social influence on a particular age group can be performed:

$$
\begin{aligned}
& \text { - for additive convolution }-\tau_{j}=\sum_{i=1}^{k+l+m+n} \rho_{i j} ; \\
& \text { - for multiplicative convolution }-\tau_{j}=1-\sum_{i=1}^{k+l+m+n}\left(1-\rho_{i j}\right) .
\end{aligned}
$$

The structural diagram of the method for assessing the total intensity of the influence of a particular institutional structure is shown in Fig. 5.

This method allows, on the basis of the proposed mathematical apparatus, to objectively determine the "formal" and "informal" influence of the respective leaders on the subjects of the regional society. The basis for this is the subjective judgments of both the subjects themselves and the results of expert assessments, opinion polls, etc.

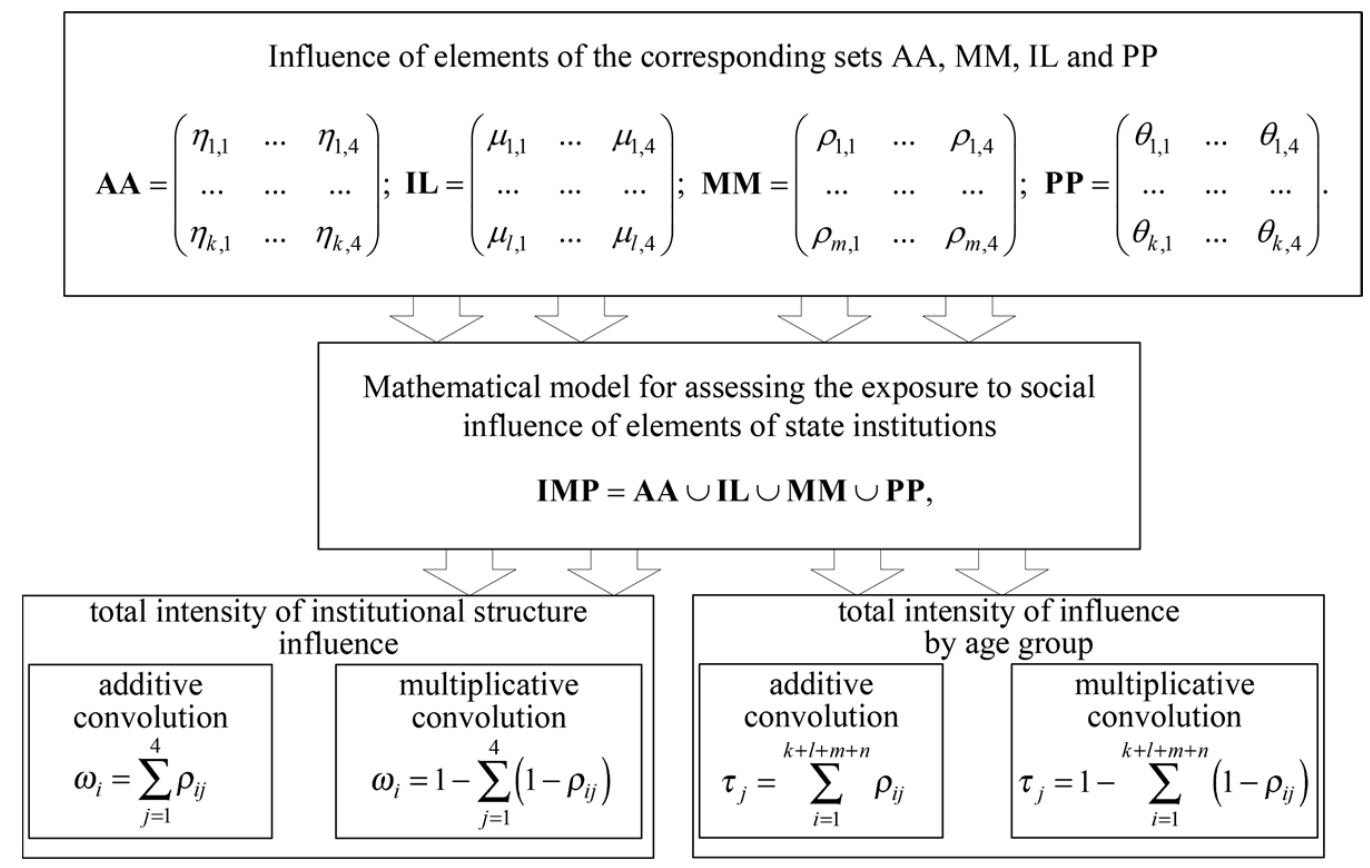

Fig. 5. Block diagram of the method for assessing the total intensity of the influence of a particular institutional structure 
5. 3. Development of a method for assessing and predicting the rating of political forces based on the mechanism of social influence

Step 7. To build a rating of political parties in the region, based on the attitude of formal and informal leaders towards them, as well as the image formed by the media, it is necessary to form a matrix for assessing political forces by the listed structures.

A mathematical model of the influence of a regional society on the formation of the rating of political forces is shown in Fig. 6 and is formally set:

$$
P R=\left(\begin{array}{lll}
\pi_{1,1} & \ldots & \pi_{1,4} \\
\ldots & \ldots & \ldots \\
\pi_{1,4} & \ldots & \pi_{k, 4} \\
\pi_{k+1,1} & \ldots & \pi_{k+1,4} \\
\ldots & \ldots & \ldots \\
\pi_{k+l, 1} & \ldots & \pi_{k+l, 4} \\
\pi_{k+l+1,1} & \ldots & \pi_{k+l+1,4} \\
\ldots & \ldots & \ldots \\
\pi_{k+l+m, 1} & \ldots & \pi_{k+l+m, 4}
\end{array}\right) .
$$

The presented model in Fig. 6 allows to predict the rating of political parties in accordance with age ranges. This approach, in contrast to the mathematical model for assessing exposure to social influence, provides a "feedback" of age groups on the attitude towards party forces.

The matrix is formed on the basis of estimates collected in the form of a table, the number of rows of which corresponds to the total number of formal and informal leaders, as well as the media (objects influencing the rating), and the number of columns corresponds to the number of political forces. Each cell of the table should contain expert assessments of the relationship of a particular structure to each of the political forces $\left(\pi_{i, j}\right)$.

The method for assessing and predicting the rating of political forces based on the mechanism of social influence is formed on the basis of the model of the influence of the regional society on the formation of the rating of political forces, as well as the corresponding convolutions.

The total score that forms the rating of a political force is obtained as a convolution of all private estimates and, depending on the selected type of convolution, has the form:

$$
\begin{aligned}
& \text { - for additive convolution }-\theta_{j}=\sum_{i=1}^{k+l+m} \pi_{i j} \text {; } \\
& \text { - for multiplicative convolution }-\theta_{j}=1-\prod_{i=1}^{k+l+m}\left(1-\pi_{i j}\right) \text {. }
\end{aligned}
$$

The structural diagram of the method and forecasting the rating of political forces is shown in Fig. 7.

Thus, the presented approach allows one to take into account the following components:

- the hierarchical structure of state institutions and their influence on the political outlook of certain social age groups;

- electorate and influence of political parties (blocs, movements), taking into account the political worldview of certain social age groups;

- influence of informal leaders not only on political parties, but also on certain social age groups;

- the possibility of changing the rating of political parties by influencing certain elements of regional/state institutions, informal leaders and/or media.

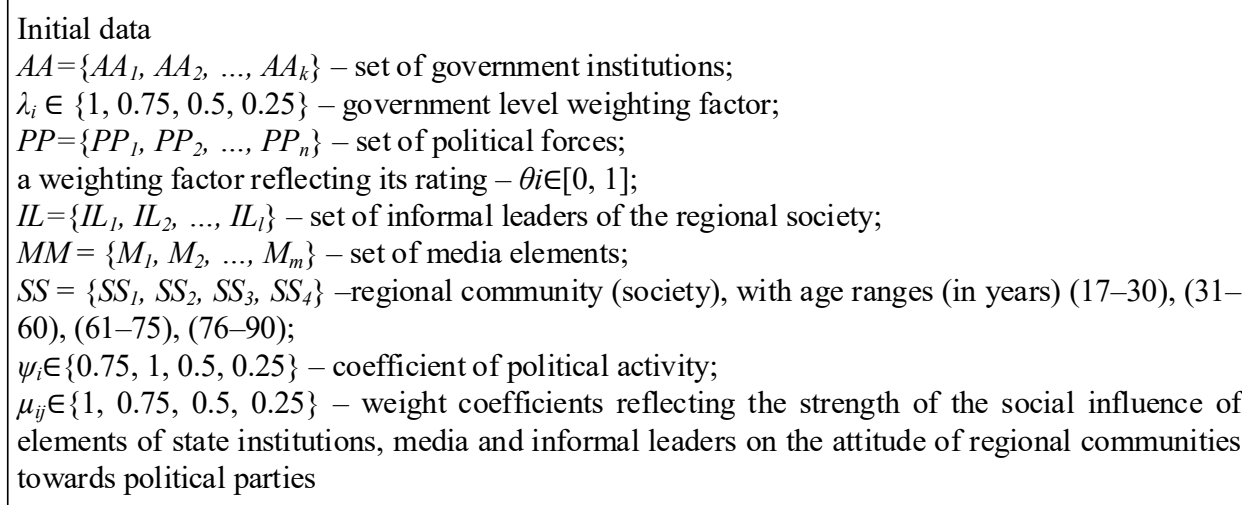

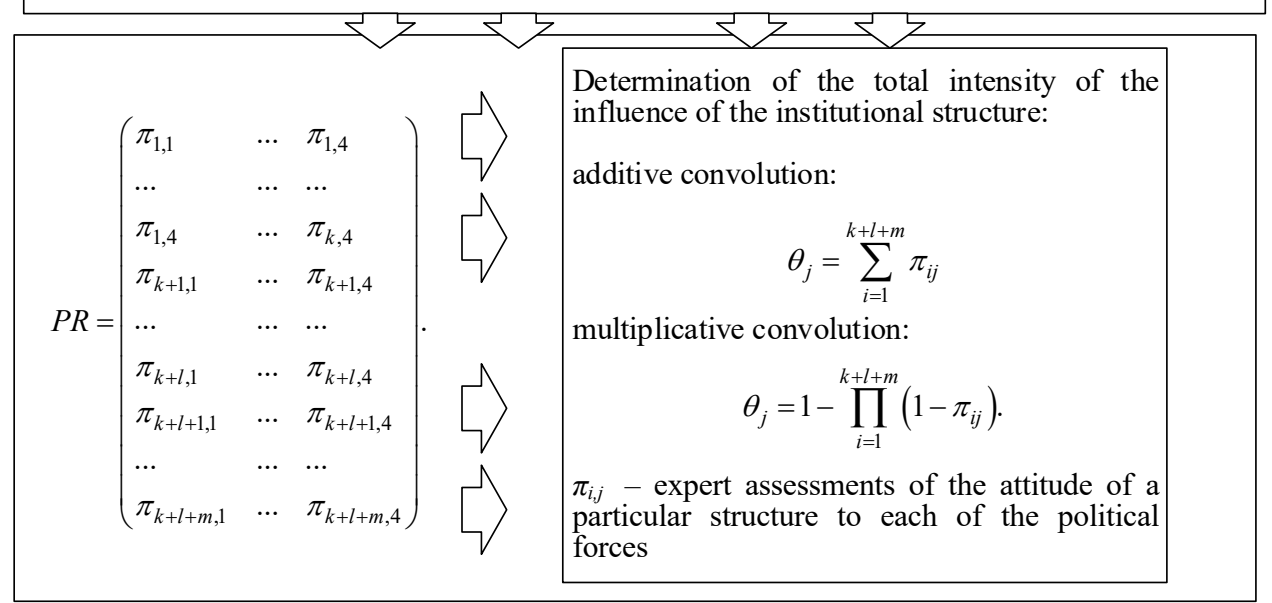

Fig. 6. Mathematical model, the influence of the regional society on the formation of the rating of political forces 


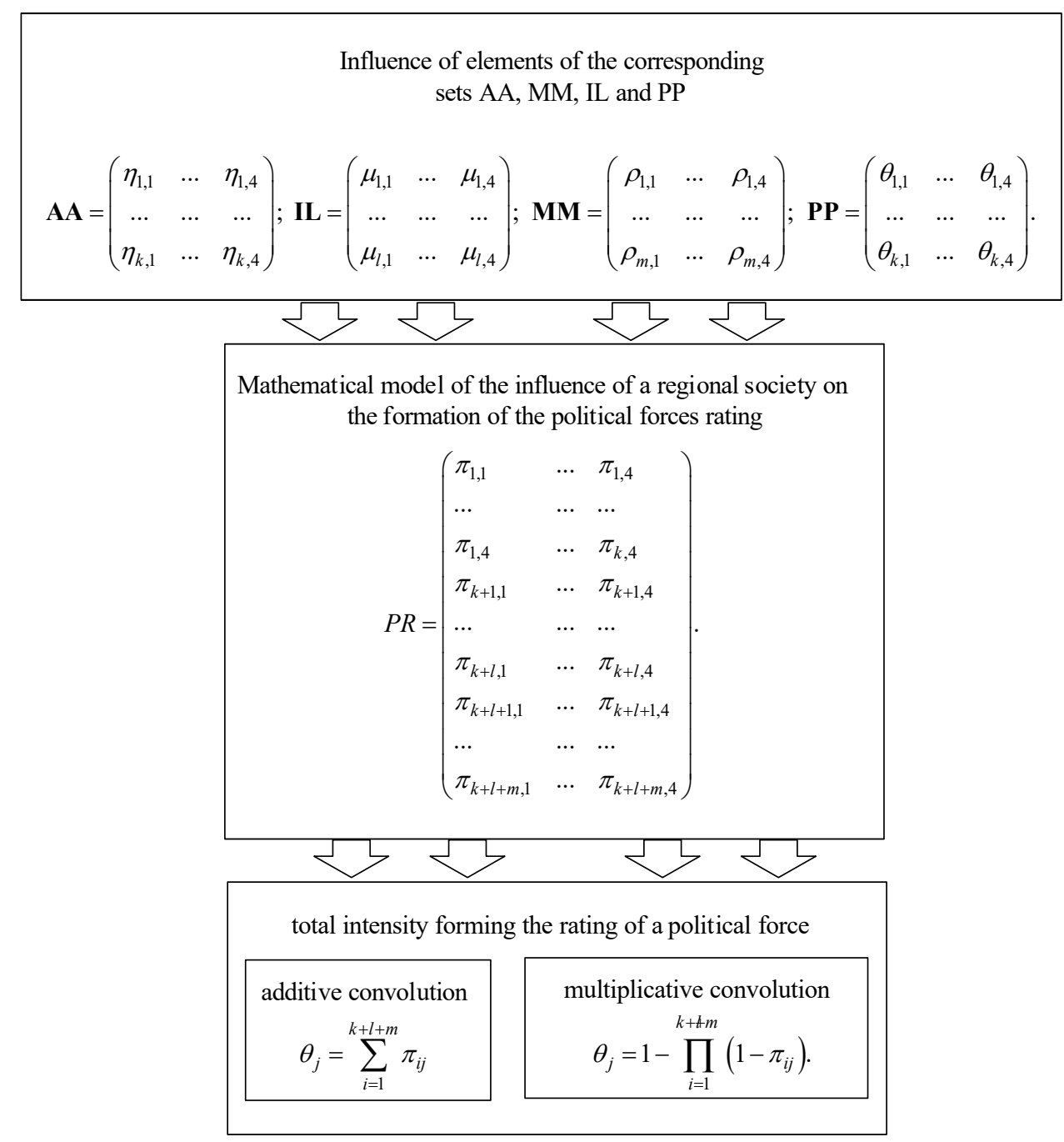

Fig. 7. Block diagram of the method and forecasting the rating of political force

\section{Discussion of the results of the study of assessing the influence of the regional society on the formation of the political forces rating}

As an example that allows to check the performance of the proposed models and methods, as well as to discuss the results obtained, let's consider a conditionally real example. Such an example will reflect the order of interaction of the presented structures and their influence on the formation of the rating of political parties and the assessment of the strength of political influence on the regional society.

Let's assume, for definiteness, there are 4 main political forces, 4 formal leaders, reflecting different levels of the administrative and state structure, 5 most influential mass media and 3 informal leaders, whose opinion is taken into account by the regional society [23].

Let's compose the $P R$ matrix. Since all estimates are dimensionless, it makes no sense to apply coefficients leading to a dimensionless unit. Also, normalizing factors are not used at this level, since they must be taken into account in the impact assessments:

$P R=\left(\begin{array}{cccc}0.9 & 0.5 & 0.7 & 0.4 \\ 0.8 & 0.4 & 0.6 & 0.7 \\ 0.6 & 0.5 & 0.4 & 0.7 \\ 0.6 & 0.7 & 0.5 & 0.9 \\ 0.7 & 0.4 & 0.3 & 0.6 \\ 0.5 & 0.9 & 0.8 & 0.7 \\ 0.4 & 0.8 & 0.5 & 0.7 \\ 0.6 & 0.7 & 0.6 & 0.9 \\ 0.9 & 0.8 & 0.5 & 0.6 \\ 0.7 & 0.3 & 0.4 & 0.9 \\ 0.8 & 0.7 & 0.4 & 0.9 \\ 0.5 & 0.8 & 0.6 & 0.7\end{array}\right)$.

When using additive convolution, let's obtain estimates for each of the 4 political forces: $P_{1}-8.0 ; P_{2}-7.5$; $P_{3}-6.3 ; P_{4}-8.7$. Thus, the rating of political forces is as follows: $P_{4} \rightarrow P_{1} \rightarrow P_{2} \rightarrow P_{1}$.

If multiplicative convolution is applied, the rating will be as follows: $P_{4} \rightarrow P_{1} \rightarrow P_{2} \rightarrow P_{1}$. As can be seen, the rating did 
not change when switching from using additive convolution to multiplicative.

A promising area of further research for constructing a rating of political forces is the use of the hierarchy analysis method. This approach will allow, in contrast to the classical method, to use the values obtained within the method to assess the spread of opinions of influencing structures, to take into account the weight of various influences on the formation of the rating. In addition, the use of the method of analyzing hierarchies will make it possible to get away from the criteria-based assessment of each of the political forces. The experts will be asked to give a comparative assessment of the attractiveness of a particular political force in the language of binary relations (i.e., in the form of pairwise comparison).

Let there be given a model of the influence of formal and informal leaders, as well as the media on the formation of the rating of political parties. On the one hand, it will be interested in the contribution of the influence of this or that subject to the general system of forming the rating of political forces. On the other hand, there is the proximity (similarity) of the influence of pairs, triples, fours, etc. of subjects on the formation of the rating.

To solve these problems, let's use the method of differentiating models. It should be noted that the method was presented to be used as a starting point for a matrix of incidents, containing as values the elements of zeros and ones, reflecting the existence of a relationship between a pair of vertices. Further, it is proposed to use this method in the case when the connections between the vertices are weighted, and the weight reflects the corresponding force of influence or the assessment of the corresponding object.

As a starting point, let's use the $P R$ matrix presented earlier.

The intensity of participation of a particular structure in the general system of social influence (as an example, the formation of a rating of political forces), let's use the concept of a frequency matrix of relations. The frequency matrix of relations is called a square matrix, where each row (column) corresponds to one or another agent influencing the processes under consideration, and the values of the elements are determined as follows:

$$
f_{i j}=\left\{\begin{array}{l}
\text { reduced sum of the joint influence } \\
\text { of } i \text {-th and } j \text {-th subjects if } i \neq j, \\
\text { total assessments of } i \text {-th subject if } i=j .
\end{array}\right.
$$

The frequency relationship matrix can be calculated as $P R^{\mathrm{T}} \times P R \rightarrow F$ (where the superscript $T$ indicates that the transpose matrix $P R$ is being used).

The constructed frequency matrix of ratios based on the initial data of the experiment is presented in Table 1.

The frequency matrix of relations is an intermediate result used to derive a derivative of the model under consideration by a predicate defined as a set of subjects of influence. The elements of the specified matrix are calculated by the formula:

$$
d_{i j}=\frac{\left(f_{i i}-f_{i j}\right)+\left(f_{i j}-f_{i j}\right)}{f_{i j}}=\frac{f_{i i}-2 \cdot f_{i j}+f_{i j}}{f_{i j}} .
$$

The matrix of derivatives constructed for example based on the model of the influence of the regional society on the formation of the rating of political forces is given in Table 2 .

Diagonal in Table 2 is zero, which indicates a zero proximity of the influence of each of the subjects with oneself. Non-diagonal elements should be interpreted as follows: the greater the value for a pair of subjects, the greater the discrepancy in the degree of influence of the subjects determining this value $\left(d_{i j}=d_{j i}\right)$. The indicated interpretation of the matrix elements makes it possible to find subjects that have a similar influence on the processes under consideration.

Let's find the element with the minimum value. This is the element $d_{4,8}=0.005$. This means that the $4^{\text {th }}$ and $8^{\text {th }}$ subjects of influence have a similar nature of influence on the processes under consideration. The next most important element will be $d_{7,12}=0.012$. The found pairs can be considered as the closest in terms of the nature of the influence and, when building an aggregated model of less complexity, can be replaced by one element with the total intensity of the influence.

Table 1

Frequency relationship matrix $f_{i j}$ for the model of the influence of the regional society on the formation of the rating of

\begin{tabular}{|c|c|c|c|c|c|c|c|c|c|c|c|c|}
\hline subjects & \multicolumn{12}{|c|}{$j$-subject } \\
\hline \multirow{12}{*}{$i$-subject } & 1.71 & 1.62 & 1.35 & 1.60 & 1.28 & 1.74 & 1.39 & 1.67 & 1.80 & 1.42 & 1.71 & 1.55 \\
\hline & 1.62 & 1.65 & 1.41 & 1.69 & 1.32 & 1.73 & 1.43 & 1.75 & 1.76 & 1.55 & 1.79 & 1.57 \\
\hline & 1.35 & 1.41 & 1.26 & 1.54 & 1.16 & 1.56 & 1.33 & 1.58 & 1.56 & 1.36 & 1.62 & 1.43 \\
\hline & 1.60 & 1.69 & 1.54 & 1.91 & 1.39 & 1.96 & 1.68 & 1.96 & 1.89 & 1.64 & 1.98 & 1.79 \\
\hline & 1.28 & 1.32 & 1.16 & 1.39 & 1.10 & 1.37 & 1.17 & 1.42 & 1.46 & 1.27 & 1.50 & 1.27 \\
\hline & 1.74 & 1.73 & 1.56 & 1.96 & 1.37 & 2.19 & 1.81 & 2.04 & 1.99 & 1.57 & 1.98 & 1.94 \\
\hline & 1.39 & 1.43 & 1.33 & 1.68 & 1.17 & 1.81 & 1.54 & 1.73 & 1.67 & 1.35 & 1.71 & 1.63 \\
\hline & 1.67 & 1.75 & 1.58 & 1.96 & 1.42 & 2.04 & 1.73 & 2.02 & 1.94 & 1.68 & 2.02 & 1.85 \\
\hline & 1.80 & 1.76 & 1.56 & 1.89 & 1.46 & 1.99 & 1.67 & 1.94 & 2.06 & 1.61 & 2.02 & 1.81 \\
\hline & 1.42 & 1.55 & 1.36 & 1.64 & 1.27 & 1.57 & 1.35 & 1.68 & 1.61 & 1.55 & 1.74 & 1.46 \\
\hline & 1.71 & 1.79 & 1.62 & 1.98 & 1.50 & 1.98 & 1.71 & 2.02 & 2.02 & 1.74 & 2.10 & 1.83 \\
\hline & 1.55 & 1.57 & 1.43 & 1.79 & 1.27 & 1.94 & 1.63 & 1.85 & 1.81 & 1.46 & 1.83 & 1.74 \\
\hline
\end{tabular}
political forces 
Table 2

Derivative matrix $d_{i j}$ for the model of the influence of the regional society on the formation of the rating of political forces

\begin{tabular}{|c|c|c|c|c|c|c|c|c|c|c|c|c|}
\hline subjects & \multicolumn{12}{|c|}{$j$-subject } \\
\hline \multirow{12}{*}{$i$-subject } & 0.000 & 0.074 & 0.200 & 0.263 & 0.195 & 0.241 & 0.338 & 0.234 & 0.094 & 0.296 & 0.228 & 0.226 \\
\hline & 0.074 & 0.000 & 0.064 & 0.107 & 0.083 & 0.220 & 0.231 & 0.097 & 0.108 & 0.065 & 0.095 & 0.159 \\
\hline & 0.200 & 0.064 & 0.000 & 0.058 & 0.034 & 0.212 & 0.105 & 0.076 & 0.128 & 0.066 & 0.074 & 0.098 \\
\hline & 0.263 & 0.107 & 0.058 & 0.000 & 0.165 & 0.092 & 0.054 & 0.005 & 0.101 & 0.110 & 0.025 & 0.039 \\
\hline & 0.195 & 0.083 & 0.034 & 0.165 & 0.000 & 0.401 & 0.256 & 0.197 & 0.164 & 0.087 & 0.133 & 0.236 \\
\hline & 0.241 & 0.220 & 0.212 & 0.092 & 0.401 & 0.000 & 0.061 & 0.064 & 0.136 & 0.382 & 0.167 & 0.026 \\
\hline & 0.338 & 0.231 & 0.105 & 0.054 & 0.256 & 0.061 & 0.000 & 0.058 & 0.156 & 0.289 & 0.129 & 0.012 \\
\hline & 0.234 & 0.097 & 0.076 & 0.005 & 0.197 & 0.064 & 0.058 & 0.000 & 0.103 & 0.125 & 0.040 & 0.032 \\
\hline & 0.094 & 0.108 & 0.128 & 0.101 & 0.164 & 0.136 & 0.156 & 0.103 & 0.000 & 0.242 & 0.059 & 0.099 \\
\hline & 0.296 & 0.065 & 0.066 & 0.110 & 0.087 & 0.382 & 0.289 & 0.125 & 0.242 & 0.000 & 0.098 & 0.253 \\
\hline & 0.228 & 0.095 & 0.074 & 0.025 & 0.133 & 0.167 & 0.129 & 0.040 & 0.059 & 0.098 & 0.000 & 0.098 \\
\hline & 0.226 & 0.159 & 0.098 & 0.039 & 0.236 & 0.026 & 0.012 & 0.032 & 0.099 & 0.253 & 0.098 & 0.000 \\
\hline
\end{tabular}

Thus, the proposed approach makes it possible, when analyzing the influence and formation of initial data (weight coefficients), to form an assessment of the influence of a regional society on the formation of a rating of political forces. The results obtained can be used to assess the influence of both formal and informal leaders in a particular regional society, taking into account their weighting coefficients of influence.

The main limitations of the proposed method is the subjectivity of the expert assessment of the weight coefficients, the corresponding communication lines (impact) on the corresponding elements of the proposed models for predicting the rating of political forces and the influence of a regional society on the formation of the rating of political forces. A further direction of research development is the formation of a software package that will automate the process of constructing a structural diagram of a regional society, the interaction of both formal and informal connections between the elements of the structure, as well as the possibility of analyzing the results offline.

\section{Conclusions}

1. The models for assessing the susceptibility to social influence of elements of state institutions, media and infor- mal leaders on regional communities in relation to political parties, as well as the influence of a regional society on the formation of a political forces rating have been developed. The proposed mathematical apparatus is intuitive and universal, which makes it possible to form the initial data for assessing both the political, social and economic situation in the regional society.

2. The proposed method for assessing the total intensity of the influence of a particular institutional structure on the basis of an expert assessment takes into account the hierarchical structure of state power, the possibilities of influence both in the vertical and horizontal planes. In addition, it takes into account both the formal aspect of influence and informal relationships between subjects at the regional level.

3. The developed method for assessing and predicting the rating of political forces based on the mechanism of social influence allows to determine the political weight of political forces in the region. The method also provides an understanding of the formation of the rating based on the analysis of the impact on the regional society of both formal and informal subjects. This approach provides an opportunity to obtain an objective assessment of the social and political situation in the region.

\section{Reference}

1. Hryshchuk, R. V., Danyk, Yu. H. (2016). Osnovy kibernetychnoi bezpeky. Zhytomyr: ZhNAEU, 636.

2. Xia, F., Ma, J. (2011). Building smart communities with cyber-physical systems. Proceedings of 1st International Symposium on From Digital Footprints to Social and Community Intelligence - SCI '11. doi: https://doi.org/10.1145/2030066.2030068

3. Guo, B., Yu, Z., Zhou, X. (2015). A Data-Centric Framework for Cyber-Physical-Social Systems. IT Professional, 17 (6), 4-7. doi: https://doi.org/10.1109/mitp.2015.116

4. Kuang, L., Yang, L. T., Liao, Y. (2020). An Integration Framework on Cloud for Cyber-Physical-Social Systems Big Data. IEEE Transactions on Cloud Computing, 8 (2), 363-374. doi: https://doi.org/10.1109/tcc.2015.2511766

5. Lin, C.-C., Deng, D.-J., Jhong, S.-Y. (2020). A Triangular NodeTrix Visualization Interface for Overlapping Social Community Structures of Cyber-Physical-Social Systems in Smart Factories. IEEE Transactions on Emerging Topics in Computing, 8 (1), 58 -68. doi: https://doi.org/10.1109/tetc.2017.2671846

6. De, S., Zhou, Y., Larizgoitia Abad, I., Moessner, K. (2017). Cyber-Physical-Social Frameworks for Urban Big Data Systems: A Survey. Applied Sciences, 7 (10), 1017. doi: https://doi.org/10.3390/app7101017

7. Bond, R. M., Fariss, C. J., Jones, J. J., Kramer, A. D. I., Marlow, C., Settle, J. E., Fowler, J. H. (2012). A 61-million-person experiment in social influence and political mobilization. Nature, 489 (7415), 295-298. doi: https://doi.org/10.1038/nature11421 
8. Jayles, B., Kim, H., Escobedo, R., Cezera, S., Blanchet, A., Kameda, T. et. al. (2017). How social information can improve estimation accuracy in human groups. Proceedings of the National Academy of Sciences, 114 (47), 12620-12625. doi: https://doi.org/10.1073/ pnas. 1703695114

9. Almaatouq, A., Noriega-Campero, A., Alotaibi, A., Krafft, P. M., Moussaid, M., Pentland, A. (2020). Adaptive social networks promote the wisdom of crowds. Proceedings of the National Academy of Sciences, 117 (21), 11379-11386. doi: https:// doi.org/10.1073/pnas.1917687117

10. Phoa, F. K. H., Weng, P. C.-Y., Chiang, Y.-S. (2016). A mathematical model on the propagation of node attributes on a social network. IAENG Transactions on Engineering Sciences. doi: https://doi.org/10.1142/9789813142725_0009

11. Kao, A. B., Berdahl, A. M., Hartnett, A. T., Lutz, M. J., Bak-Coleman, J. B., Ioannou, C. C. et. al. (2018). Counteracting estimation bias and social influence to improve the wisdom of crowds. Journal of The Royal Society Interface, 15 (141), 20180130. doi: https:// doi.org/10.1098/rsif.2018.0130

12. Lorenz, J., Rauhut, H., Schweitzer, F., Helbing, D. (2011). How social influence can undermine the wisdom of crowd effect. Proceedings of the National Academy of Sciences, 108 (22), 9020-9025. doi: https://doi.org/10.1073/pnas.1008636108

13. Jayles, B., Sire, C., Kurvers, R. H. J. M. (2021). Impact of sharing full versus averaged social information on social influence and estimation accuracy. Journal of The Royal Society Interface, 18 (180), 20210231. doi: https://doi.org/10.1098/rsif.2021.0231

14. Madirolas, G., de Polavieja, G. G. (2015). Improving Collective Estimations Using Resistance to Social Influence. PLOS Computational Biology, 11 (11), e1004594. doi: https://doi.org/10.1371/journal.pcbi.1004594

15. Parthasarathy, S., Ruan, Y., Satuluri, V. (2011). Community Discovery in Social Networks: Applications, Methods and Emerging Trends. Social Network Data Analytics, 79-113. doi: https://doi.org/10.1007/978-1-4419-8462-3_4

16. Sun, J., Tang, J. (2011). A Survey of Models and Algorithms for Social Influence Analysis. Social Network Data Analytics, 177-214. doi: https://doi.org/10.1007/978-1-4419-8462-3_7

17. Anagnostopoulos, A., Kumar, R., Mahdian, M. (2008). Influence and correlation in social networks. Proceeding of the 14th ACM SIGKDD International Conference on Knowledge Discovery and Data Mining - KDD 08. doi: https://doi.org/ $10.1145 / 1401890.1401897$

18. Goyal, A., Bonchi, F., Lakshmanan, L. V. S. (2010). Learning influence probabilities in social networks. Proceedings of the Third ACM International Conference on Web Search and Data Mining - WSDM '10. doi: https://doi.org/10.1145/1718487.1718518

19. Xiang, R., Neville, J., Rogati, M. (2010). Modeling relationship strength in online social networks. Proceedings of the 19th International Conference on World Wide Web - WWW '10. doi: https://doi.org/10.1145/1772690.1772790

20. Scripps, J., Tan, P.-N., Esfahanian, A.-H. (2009). Measuring the effects of preprocessing decisions and network forces in dynamic network analysis. Proceedings of the 15th ACM SIGKDD International Conference on Knowledge Discovery and Data Mining KDD '09. doi: https://doi.org/10.1145/1557019.1557102

21. Tang, L., Liu, H. (2009). Relational learning via latent social dimensions. Proceedings of the 15th ACM SIGKDD International Conference on Knowledge Discovery and Data Mining - KDD '09. doi: https://doi.org/10.1145/1557019.1557109

22. Yevseiev, S., Ponomarenko, V., Laptiev, O., Milov, O., Korol, O., Milevskyi, S. et. al. (2021). Synergy of building cybersecurity systems. Kharkiv: PC TECHNOLOGY CENTER, 188. doi: https://doi.org/10.15587/978-617-7319-31-2

23. Pozacherhovi vybory narodnykh deputativ Ukrainy 21 lypnia 2019 roku. Ofitsiynyi sait «Tsentralnoi vyborchoi komisii Ukrainy». Available at: https://www.cvk.gov.ua/vibory_category/vibori-narodnih-deputativ-ukraini/pozachergovi-vibori-narodnihdeputativ-ukraini-21-lipnya-2019-roku.html 